\title{
Magnetism-driven dislocation dissociation, cross slip, and mobility in NiAl
}

\author{
Yi Zhao \\ Department of Physics, Tsinghua University, Beijing 100084, People's Republic of China \\ and Department of Physics and Astronomy, California State University Northridge, Northridge, California 91330-8268, USA \\ Chongyu Wang \\ Department of Physics, Tsinghua University, Beijing 100084, People's Republic of China \\ Gang Lu \\ Department of Physics and Astronomy, California State University Northridge, Northridge, California 91330-8268, USA
}

(Received 7 June 2010; published 20 August 2010)

\begin{abstract}
We have examined the interplay between magnetism and dislocation core properties in NiAl alloys using quantum mechanics/molecular mechanics simulations. We observe the magnetism-driven site preference of $\mathrm{Fe}$ and Co impurities at the dislocation core. When occupying the Ni sublattice, Fe and Co impurities are found to induce spontaneous dislocation cross slip. When occupying the Al sublattice, the impurities render the originally undissociated dislocation to split into two partials. Finally we observe the magnetism-driven dislocation mobility when Fe impurity occupies the Al sublattice, which has also been discovered experimentally. The magnetic interaction between the impurities and the host atoms is responsible for the significant changes in the dislocation core structure and mobility.
\end{abstract}

DOI: 10.1103/PhysRevB.82.060404

PACS number(s): 75.30.Hx, 61.72.Lk, 61.72.Nn, 71.20.Lp

It has been shown experimentally that an applied magnetic field can change the plastic behavior of crystalline materials, a phenomenon known as magnetoplasticity. ${ }^{1,2}$ In a series of theoretical papers, Molotskii and Fleurov have explained the phenomenon by assuming that a dangling bond of a paramagnetic impurity forms a radical pair with a dangling bond of the dislocation core. They showed that the applied magnetic field could then induce transitions between different spin states of such pairs characterized by different binding energies, and thus facilitate dislocation depinning from the impurity and enhance the crystal plasticity. ${ }^{3}$ Despite its physical significance, the magnetoplasticity phenomenon becomes less relevant in most applications where materials deform in the absence of an applied magnetic field. However, a recent work combining theory and experiments ${ }^{4,5}$ has shown that even in the absence of the magnetic field, magnetism plays an important role in the mechanical response of materials. In particular, it is discovered that $3 d$ transitionmetal impurities (Fe, $\mathrm{Co}, \mathrm{Ni}, \mathrm{Cr}$, etc.) could profoundly change the mechanical properties of $\mathrm{B} 2-\mathrm{NiAl}$ intermetallic alloys; the origin of the impurity-induced solid solution softening in these alloys is due to the magnetic interaction between the impurities and the host atoms. ${ }^{6}$ The experimentally observed softening can only be reproduced by densityfunctional theory (DFT) calculations if the magnetic interaction (or spin polarization) is turned on. Similarly, the site preference and lattice spacing changes would switch sign if the spin polarization is turned off in the DFT calculations. These results along with the magnetoplasticity effect, highlight the important role that magnetism plays in the mechanical response of materials, illustrating the cross fertilization between two disciplines, magnetism and mechanical properties.

B2 intermetallic NiAl alloys have attracted a lot of attention recently owing to their potential applications as hightemperature structural materials. One of the common prac- tices to improve the mechanical properties of materials is alloying. For example, introducing substitutional elements including $\mathrm{Fe}, \mathrm{Co}, \mathrm{Ni}$, etc., in $\mathrm{NiAl}$ has been shown to enhance the strength and ductility of the alloy. These impurities can strongly interact with lattice defects, in particular, dislocations; it is through this interaction that the impurities affect the mechanical properties of the material. On the other hand, it is well known that the dangling bonds at the defect core could introduce localized electronic states and magnetic moments. ${ }^{3,7,8}$ In some cases, these defects could eventually lead to magnetic ordering. Therefore, the interplay between magnetism and lattice defects-lattice defects could give rise to magnetism and magnetism could affect the properties of lattice defects, offers an intriguing perspective that could be useful in the design of novel materials.

In this Rapid Communication, we explore the interplay between magnetism and dislocation properties in NiAl in the absence of magnetic field. Our first-principles based quantum mechanics/molecular mechanics (QM/MM) calculations show that by substituting $\mathrm{Ni}$ atom at the dislocation core, $\mathrm{Fe}$ and Co impurities can lead to spontaneous dislocation cross slip. On other hand, by replacing $\mathrm{Al}$ atom at the core, Fe, Co, and $\mathrm{Ni}$ impurities can result in dislocation dissociation into partials. In addition, we find that the site preference of Fe and Co impurities at the dislocation core is magnetism driven. Finally we observe magnetism-enhanced dislocation mobility when the $\mathrm{Al}$ atom is replaced by $\mathrm{Fe}$ at the dislocation core. Our calculations unambiguously demonstrate the connections between magnetism and the mechanical properties of NiAl alloys.

In the QM/MM method, the simulated system is partitioned into two regions (see Fig. 1). The region I is treated with the quantum-mechanical density-functional theory (DFT) and contains the dislocation core; the region II on the other hand is treated with the empirical embedded-atommethod (EAM) (Ref. 9) and contains the rest of system, in- 


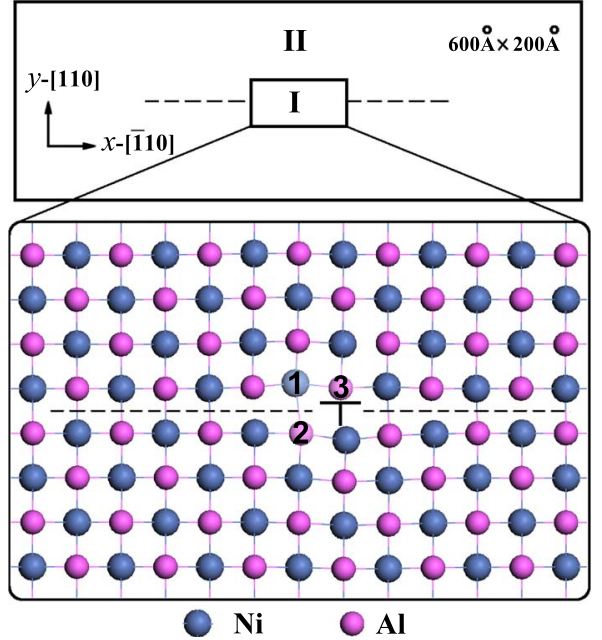

FIG. 1. (Color online) Schematic partition of the computational model into region I and II with a blown-up view of region I. The slip plane is denoted by a dashed line. The substitutional sites are labeled by numbers from 1 to 3 .

cluding the long-range elastic field of the dislocation. The two regions are mechanically coupled and the interaction energy is computed by EAM. Therefore, the total energy of the system can be written as

$$
E_{\mathrm{T}}=E_{\mathrm{I}, \mathrm{II}}^{\mathrm{EAM}}+E_{\mathrm{I}}^{\mathrm{DFT}}-E_{\mathrm{I}}^{\mathrm{EAM}}
$$

where the three terms on the right-hand side represent the energy of the entire system treated with EAM, the energy of region I treated with DFT, and the energy of region I treated with EAM, respectively. The technical details of the method along with error analysis can be found in Ref. 10 . We use the VASP package ${ }^{11}$ as the DFT approach with the projected augmentation wave method ${ }^{12}$ for the pseudopotentials under the generalized gradient approximation. ${ }^{13}$ The screw dislocation with the Burgers vector [001] which plays a central role in the plastic deformation of NiAl is studied in the paper. ${ }^{14}$ The entire system measures $600 \AA \times 200 \AA \times 2.894 \AA$ in $x, y$, and $z$ directions, respectively. The fixed boundary conditions are applied along $x$ and $y$ directions with the boundary displacement determined by the elastic solution of the dislocation. The dislocation line is along $z$ axis in which the periodical boundary condition is applied. Therefore, we are simulating a row (or rows) of impurity atoms interacting with a straight dislocation. The impurity atoms are separated by $2.894 \AA$ apart in the $z$ direction. In this Rapid Communication, we are interested to explore magnetism-driven site preference, dissociation, cross slip and mobility of a straight dislocation segment. The slip plane $x o z$ is (110). The entire system consists of 28714 atoms with 104 atoms in region I shown schematically in Fig. 1. The force convergence criterion is $0.02 \mathrm{eV} / \AA$ for all atoms.

Pure NiAl dislocation core structure. The dislocation core structure can be characterized by either the relative displacement density plot or the differential displacement field plot, shown in Figs. 2(a) and 2(c), respectively, for the pure NiAl screw dislocation. The former is defined as the derivative of the relative displacement in the $z$ direction across the slip

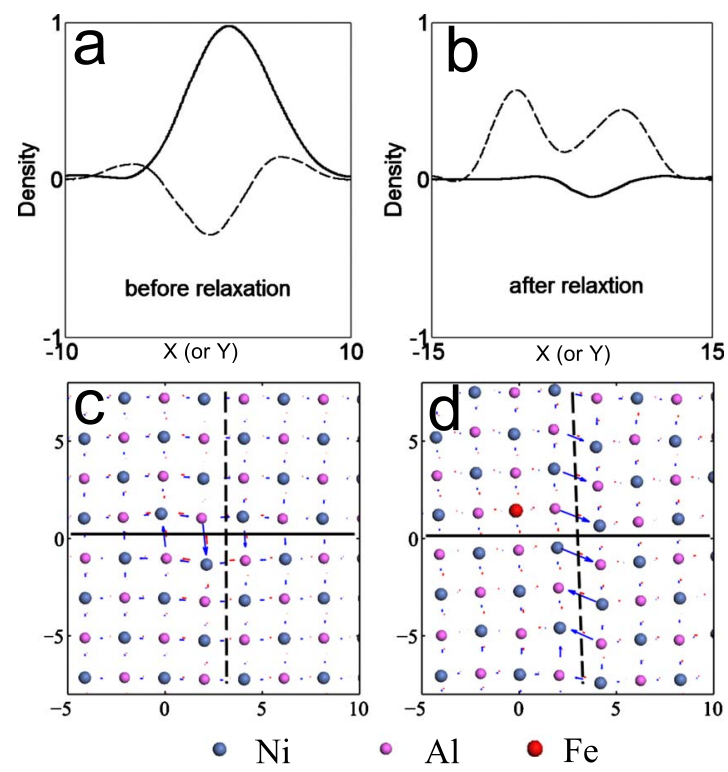

FIG. 2. (Color online) Cross slip represented by the dislocation displacement density plots (upper panel) and the differential displacement plots (lower panel). (a) Before the relaxation: displacement density in the (110) primary slip plane is shown in the solid curve and in (110) cross-slip plane is shown in the dashed curve. (b) After the relaxation: the caption is the same as (a). (c) Before the relaxation: the $z$ displacement is shown in blue and the $y$ displacement is shown in red. The magnitude of the differential displacement is proportional to the length of the arrow. The solid and dashed lines indicate the primary and cross-slip plane, respectively. The horizontal and vertical axes (in $\AA$ ) correspond to $x$ and $y$ direction, respectively. (d) After the relaxation: the caption is the same as (c) except the $x$ displacement instead of the $y$ displacement is shown (in red).

plane as a function of $x$. The latter is defined as the relative displacement between a pair of atoms joined by an arrow. The screw dislocation appears to be nondissociated with a single peak in the density plot shown in Fig. 2(a). In addition, the screw dislocation core is found to be planar, i.e., the displacement is primarily confined within the slip plane. The in-plane displacement along the $z$ direction (blue arrow) is much larger than the out-of-plane displacement along $y$ the direction (red arrow).

Magnetism-driven site preference. Site occupation of substitutional impurities is an important factor that influences the properties of multicomponent alloys. ${ }^{15-17}$ To examine the site preference at the dislocation core, we calculate the cohesive energy of the dislocation by substituting $\mathrm{Fe}, \mathrm{Co}$, and $\mathrm{Ni}$ impurities for either $\mathrm{Ni}$ or $\mathrm{Al}$ sublattice, whose positions are labeled by 1 and 2 in Fig. 1, respectively. In the following, we use symbol $\mathrm{A}(\mathrm{B})$ to represent the substitution of host atom B by impurity $\mathrm{A}$. The cohesive energy is relative to the pure NiAl dislocation and a negative cohesive energy indicates that the dislocation is energetically more stable with the impurities; however, this does not necessarily mean there is a binding between the dislocation and the impurities. Both the spin polarized and the nonspin-polarized DFT calculations are performed for region I in which the energy difference signifies the importance of magnetic interaction in determin- 
TABLE I. Cohesive energy with respect to the pure system. For each impurity the results from the spin polarized and the nonspinpolarized (in parenthesis) calculations are shown for comparisons. $\Delta E$ is the site energy difference for occupying the $\mathrm{Ni}$ and $\mathrm{Al}$ sites. $\Delta E_{\text {spin }}$ is the spin-induced site energy difference. The energy unit is electron volt.

\begin{tabular}{lccc}
\hline \hline & \multicolumn{3}{c}{ Site } \\
\cline { 2 - 4 } Impurity & $\mathrm{Fe}$ & $\mathrm{Co}$ & $\mathrm{Ni}$ \\
\hline $\mathrm{Ni}$ & $-0.73(-0.82)$ & $-1.29(-1.07)$ & $0(0)$ \\
$\mathrm{Al}$ & $-1.00(-0.37)$ & $-1.25(-1.10)$ & $-0.85(-0.80)$ \\
$\Delta E$ & $0.27(-0.45)$ & $-0.04(0.03)$ & $0.85(0.80)$ \\
$\Delta E_{\text {spin }}$ & 0.72 & -0.08 & 0.05 \\
\hline \hline
\end{tabular}

ing the site preference. From Table I, it is found that in all cases, the presence of the impurities lowers the energy of the dislocation. It is observed that the Fe impurity prefers to occupy the Al site whereas Co prefers the Ni site. More interestingly, the site preference of Fe appears to be magnetism driven-the spin-on and spin-off calculations predict the opposite site preference, with a very large energy difference $(0.72 \mathrm{eV})$. This energy difference is due to the fact that there is a strong ferromagnetic interaction between Fe and the neighboring $\mathrm{Ni}$ atoms, which is absent when the spin is turned off. However, this spin-induced energy difference for $\mathrm{Co}$ and $\mathrm{Ni}$ impurities is much smaller. Spin polarization seems to enhance the binding of the impurities at the dislocation core with the exception of $\mathrm{Fe}(\mathrm{Ni})$. We find that for $\mathrm{Fe}(\mathrm{Al})$ and $\mathrm{Co}(\mathrm{Al})$, a magnetic moment of $2.8 \mu_{B}$ and $1.4 \mu_{B}$ is developed on Fe and Co site, respectively. There is some small induced magnetic moment $\left(0.1 \mu_{B}\right)$ on each nearest-neighbor Ni atom. No magnetic moment is observed when the impurities occupy the Ni sublattice.

Impurity-induced spontaneous cross slip. Cross slip is an activated process with an energy barrier that could be overcome by either thermal excitation or external stress. To examine the impurity effect on dislocation cross slip, we introduce an Fe impurity substituting a $\mathrm{Ni}$ host atom at the dislocation core, labeled by 1 in Fig. 1. In the absence of the impurity, the dislocation is spread onto the primary (110) plane as indicated by a horizontal line in Fig. 2. There is a negligible displacement field in the perpendicular slip plane (110), hereafter referred to as the cross-slip plane indicated by a vertical dashed line in Fig. 2. We should note that although the (110) and ( $\overline{1} 10)$ planes are crystallographically equivalent, once the dislocation is introduced in the (110) plane, the symmetry is broken and the dislocation is spread onto the (110) plane. In other words, there is an energy barrier for the dislocation to cross slip to the $(\overline{1} 10)$ plane in pure $\mathrm{NiAl}$. However, when the Fe impurity is introduced, we find that the cross slip takes place spontaneously, as illustrated in Fig. 2: there is a negligible displacement field left in the (110) plane and the most of the displacement field has transferred to the cross-slip plane $(\overline{1} 10)$. Moreover, the crossslipped dislocation subsequently dissociates into two partial dislocations indicated by the double peak in the density plot.
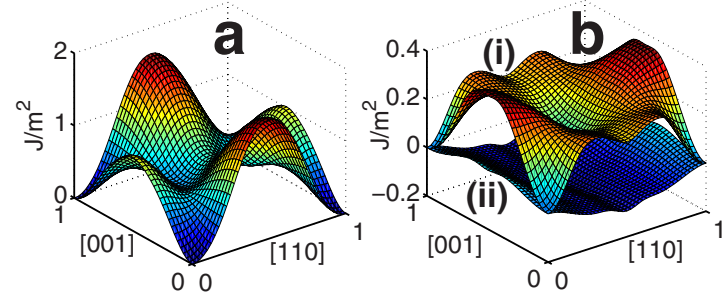

FIG. 3. (Color online) (a) The $\gamma$ surface of the pure bulk NiAl. (b) $\Delta \gamma$ of cases (i) and (ii) with respect to the pure system; see text.

The partial dislocations here are of the generalized type ${ }^{18}$ and they are not necessarily associated with the stable stacking fault as the Shockley partials are. More importantly, the dislocation moves away from the Fe impurity. The spontaneous cross slip can be understood from the $\gamma$ surface $^{19}$ of the alloys. In particular, we focus on two configurations: (i) the Fe impurity is placed right above the slip plane which corresponds to the situation before the cross slip takes place. In this case, the slip plane is (110). (ii) The Fe impurity is placed at one atomic layer away from the slip plane which corresponds to the situation after the cross slip takes place. In this case, the slip plane is $(\overline{1} 10)$. We find that in the case (i), the $\gamma$ energy has increased considerably for all planar displacements on the (110) plane comparing to the pure system. As a result, the dislocation core energy increases significantly. On the other hand, in the case (ii), the $\gamma$ energy decreases. The relative $\gamma$ energy with respect to the pure system is shown in Fig. 3(b) for both (i) and (ii). The $\gamma$ energy difference between (i) and (ii) is essentially the driving force for the spontaneous dislocation cross slip, i.e., the dislocation tries to avoid the Fe impurity by changing its slip plane from (110) to $(\overline{1} 10)$. The same phenomenon is also observed for $\mathrm{Co}(\mathrm{Ni})$.

Impurity-induced dislocation dissociation. Next we examine the dislocation core structure when an Al host atom is replaced by the magnetic impurities. We find that when $\mathrm{Al}$ is substituted by $\mathrm{Fe}, \mathrm{Co}$, and $\mathrm{Ni}$ impurities at the dislocation core, the original undissociated dislocation splits into two partial dislocations. An example of the dissociated core structure is shown in Fig. 4(a) for $\mathrm{Co}(\mathrm{Al})$ labeled by 2 in Fig. 1. The two partials are separated approximately $9 \AA$ apart. Once again the impurity-induced dissociation can be understood from the $\gamma$ surface shown in Fig. 4(b). There is a significant reduction in $\gamma$ energy in [ 110$]$ direction in the presence of the Co impurity, which renders the displacement along the $[\overline{1} 10]$ direction energetically favorable. Therefore, instead of slipping along [001]-the Burgers vector direction, the dislocation also slips "sideways" along [110], resulting a dissociation of the dislocation. This splitting mechanism is similar to fcc materials. ${ }^{20}$ There is a ferromagnetic interaction between $\mathrm{Co}$ and the neighboring $\mathrm{Ni}$ atoms; a magnetic moment of $1.4 \mu_{B}$ is developed on the Co atom and $0.1 \mu_{B}$ on the nearest-neighbor $\mathrm{Ni}$ atoms. The same dissociation behavior is also found for the $\mathrm{Ni}(\mathrm{Al})$ system in which the dislocation core becomes dissociated and wider. These results are consistent with the experimental observations. ${ }^{21}$

Magnetism-driven dislocation mobility. Dislocation mo- 

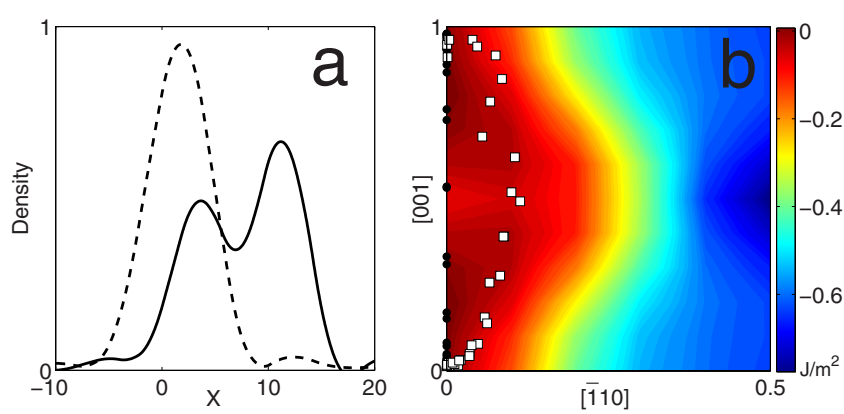

FIG. 4. (Color online) Dislocation density and $\gamma$ energy of the $\mathrm{Co}(\mathrm{Al})$ system. (a) Dislocation density of the pure system (dashed line) and the $\mathrm{Co}(\mathrm{Al})$ system (solid line). (b) The projection of $\Delta \gamma$ the $\gamma$ energy of the $\mathrm{Co}(\mathrm{Al})$ system minus and the $\gamma$ energy of the pure system onto the slip plane. The displacement field of the dislocation core atoms is superimposed on the $\gamma$ energy projection.

bility is often characterized by its Peierls stress. Here, we focus on the $\mathrm{Fe}(\mathrm{Al})$ system. The location of the impurity is labeled by 3 in Fig. 1. A shear strain along the $z$ axis is applied to the top and bottom surface of the simulation box in stepwise increments. For each increment of $2.5 \times 10^{-3}$, the dislocation is fully relaxed. The dislocation is considered moved when the center of the dislocation, the peak of the dislocation density, translates by more than one lattice spacing in $[\overline{1} 10]$ direction. We find that the Peierls stress for the dislocation in the absence and presence of the Fe impurity is $900 \mathrm{MPa}$ and $600 \mathrm{MPa}$, respectively. Thus the dislocation mobility is increased significantly; this is consistent with the experimental observation that $\mathrm{Fe}$ can reduce the hardness of Ni-rich $\mathrm{NiAl}$ alloy ${ }^{22}$ (Fe is known to occupy Al sublattice in Ni-rich condition). Moreover, the enhanced dislocation mobility appears to be magnetism driven. When the spin polarization is turned off in the DFT calculation, the dislocation core narrows and it cross slips to the (110) plane. This is because when the spin is turned off, the ferromagnetic interaction between $\mathrm{Fe}$ and $\mathrm{Ni}$ is no longer possible, thus $\mathrm{Fe}$ bonds with the neighboring $\mathrm{Al}$ atoms instead. As a result, some $\mathrm{Al}$ atoms are attracted to the Fe impurity and the dislocation core becomes narrower.

In summary, we have demonstrated the importance of magnetic interactions in changing dislocation core properties. We find the magnetism-driven site preference of $\mathrm{Fe}$ and Co impurities at the dislocation core. When occupying the $\mathrm{Ni}$ sublattice, Fe and Co impurities induce spontaneous dislocation cross slip. When occupying the Al sublattice, the impurities render the undissociated dislocation in pure $\mathrm{NiAl}$ to split into two partials. We observe the magnetism-driven dislocation mobility when Fe impurity occupies the Al sublattice, which is consistent with the experimental results. Finally, the observed magnetic interaction between the dislocation core and the impurities confirms the hypothesis of the magnetoplasticity theory.

This work was supported by NSF under Grants No. DMR-0611562 and No. DMR-0958596 and DOE under Grant No. DE-FC02-06ER25791.
${ }^{1}$ V. Ya. Kravchenko, Pisma Zh. Eksp. Teor. Fiz. 12, 551 (1970) [JETP Lett. 12, 391 (1970)].

${ }^{2}$ M. I. Molotskii, Fiz. Tverd. Tela (Leningrad) 33, 3112 (1991) [Sov. Phys. Solid State 33, 1760 (1991)].

${ }^{3}$ M. Molotskii and V. Fleurov, Phys. Rev. Lett. 78, 2779 (1997); J. Phys. Chem. B 104, 3812 (2000); Philos. Mag. Lett. 73, 11 (1996), and references therein.

${ }^{4}$ C. T. Liu et al., Prog. Mater. Sci. 52, 352 (2007).

${ }^{5}$ C. T. Liu, C. L. Fu, L. M. Pike, and D. S. Easton, Acta Mater. 50, 3203 (2002).

${ }^{6}$ P. Lazar and R. Podloucky, Intermetallics 17, 675 (2009).

${ }^{7}$ P. O. Lehtinen, A. S. Foster, Y. Ma, A. V. Krasheninnikov, and R. M. Nieminen, Phys. Rev. Lett. 93, 187202 (2004).

${ }^{8}$ M. P. López-Sancho, F. de Juan, and M. A. H. Vozmediano, Phys. Rev. B 79, 075413 (2009).

${ }^{9}$ Y. Mishin, M. J. Mehl, and D. A. Papaconstantopoulos, Phys. Rev. B 65, 224114 (2002).

${ }^{10}$ Y. Liu, G. Lu, Z. Chen, and N. Kioussis, Modell. Simul. Mater. Sci. Eng. 15, 275 (2007).
${ }^{11}$ G. Kresse and J. Hafner, Phys. Rev. B 49, 14251 (1994).

${ }^{12}$ P. E. Blöchl, Phys. Rev. B 50, 17953 (1994).

${ }^{13}$ J. P. Perdew, K. Burke, and M. Ernzerhof, Phys. Rev. Lett. 77, 3865 (1996).

${ }^{14}$ P. Gumbsch and R. Schroll, Intermetallics 7, 447 (1999).

${ }^{15}$ C. Jiang, Acta Mater. 55, 4799 (2007).

${ }^{16}$ M. Balasubramanian, D. M. Pease, J. I. Budnick, T. Manzur, and D. L. Brewe, Phys. Rev. B 51, 8102 (1995).

${ }^{17}$ S. Özcan, T. Khmelevska, S. Khmelevskyi, and P. Mohn, Intermetallics 17, 441 (2009).

${ }^{18}$ V. Vitek and F. Kroupa, Philos. Mag. 19, 265 (1969).

${ }^{19}$ V. Vitek, Cryst. Lattice Defects 5, 1 (1974).

${ }^{20}$ G. Lu, N. Kioussis, V. V. Bulatov, and E. Kaxiras, Phys. Rev. B 62, 3099 (2000).

${ }^{21}$ I. Baker P. Nagpal, F. Liu and P. R. Munroe, Acta Metall. Mater. 39, 1637 (1991).

${ }^{22}$ M. F. Savage, R. Srinivasan, M. S. Daw, T. Lograsso, and M. J. Mills, Mater. Sci. Eng., A 258, 20 (1998). 